\title{
Caracterização e quantificação dos resíduos perfurocortantes gerados por diabéticos do município de Umuarama, PR, Brasil
}

\author{
Characterization and quantification of perforating waste generated \\ by diabetics in Umuarama, Paraná, Brazil
}

Luciana Nunes dos Santos (https://orcid.org/0000-0003-2147-5315) ${ }^{1}$

Juliana Bueno Ruiz (https://orcid.org/0000-0003-0104-8359) ${ }^{1}$

${ }^{1}$ Departamento de Tecnologia, Universidade Estadual de Maringá. Av. Ângelo Moreira da Fonseca 1800, Parque Danielle. 87506-370 Umuarama PR Brasil. eng.ambiental.lununes@ gmail.com

\begin{abstract}
The scope of this research was to characterize and quantify the perforating waste produced domestically by people with diabetes mellitus (DM), as well as to analyze the discard and disposal practices for these materials. The data collection was performed through a questionnaire applied to primary care patients. The sample consisted of 149 people, the majority of whom were elderly and with a low level of schooling. Regarding the generation of waste, $88.64 \%$ of the deponents declared the use of one syringe per day, performing 1 to 4 applications. The reuse of the material was reported by $66.67 \%$ of the declarants. After being used, the waste was stored - with higher prevalence - in plastic bags (20.8\%), plastic bottles, clothes softener and mayonnaise containers, etc. (46.98\%). Ordinary household waste was the main disposal site for the perforating waste. Furthermore, it was revealed that those people who did not receive instructions regarding appropriate waste disposal discarded their waste incorrectly. Based on the results, the conclusion drawn is that the diabetics who participated in this study fail to discard and dispose of the perforating waste appropriately due to the lack of adequate guidelines and public programs.
\end{abstract}

Key words Diabetes mellitus, Health service waste, Syringes
Resumo A pesquisa teve como objetivo caracterizar e quantificar os resíduos perfurocortantes produzidos no domicílio de pessoas com diabetes mellitus (DM), bem como analisar as práticas de descarte desse material. A coleta de dados foi realizada por meio de um questionário aplicado aos pacientes da Atenção Primária. A amostra foi composta por 149 pessoas, das quais a maioria era idosa e com baixo nivel de escolaridade. No que tange à geração dos resíduos, $88,64 \%$ dos depoentes atestaram utilizar uma seringa por dia, realizando de 1 a 4 aplicações. $O$ reuso do material foi relatado por $66,67 \%$ dos investigados. Depois de utilizados, os resíduos eram armazenados - com maior prevalência - em sacolas plásticas (20,80\%), garrafas PET, frascos de amaciante/ maionese, etc. $(46,98 \%)$. O lixo doméstico comum foi o principal local de descarte dos perfurocortantes. Ademais, verificou que aqueles que não receberam informações sobre o manejo dos resíduos os desprezam de maneira incorreta. Diante dos resultados, pode-se concluir que os diabéticos que participaram desse estudo possuem práticas inadequadas de acondicionamento e descarte dos perfurocortantes em decorrência da ausência de orientações e de programas públicos.

Palavras-chave Diabetes Mellitus, Resíduos de Serviços de Saúde, Seringas 


\section{Introdução}

Os Resíduos de Serviço de Saúde (RSS) são gerados em diversas atividades de atendimento à saúde humana ou animal, incluindo serviços de assistência domiciliar, e podem ser classificados em cinco grupos dispostos de A a E. Os resíduos pertencentes ao grupo E são considerados resíduos perfurocortantes ou escarificantes e são compostos pelas lâminas de barbear, agulhas, ampolas de vidro, lancetas, espátulas, e outros similares ${ }^{1}$.

Frequentemente, esses materiais (contaminados ou não) são produzidos por setores de saúde. Todavia, eles também são gerados em ambiente domiciliar devido à presença de pessoas que fazem o uso de medicamentos injetáveis. Desse modo, acabam dificultando o gerenciamento de resíduos, pois podem se misturar ao lixo comum ou ao material reciclável.

O maior grupo representativo entre os indivíduos produtores de RSS em residência são os portadores de Diabetes Mellitus (DM)2. A terapêutica adotada para a doença inclui o monitoramento intensivo com quatro medidas diárias de glicemia capilar e aplicação de insulina fracionada de três vezes ou mais ao dia, o que implica na aquisição de um grande número de seringas ${ }^{3}$.

Para os estabelecimentos de saúde o descarte dos resíduos escarificantes é respaldado pela Agência Nacional de Vigilância Sanitária (ANVISA) - órgão responsável pela regulamentação do descarte dos RSS - a qual exige através da Resolução da Diretoria Colegiada (RDC) no 306/04 que as unidades disponham do Plano de Gerenciamento de Resíduos de Serviço de Saúde (PGR$\mathrm{SS})^{4}$. No entanto, para a população que gera esse material em ambiente domiciliar não há legislação. Devido à omissão legal e ausência de programas de gerenciamento, muitas pessoas acabam descartando seus resíduos em locais impróprios.

Em relação aos prejudicados pela disposição irregular há dois grupos que devem ser destacados, sendo o primeiro composto por aqueles que não possuem coleta regular do lixo, pois acabam descartando os resíduos em torno da área em que vivem. O segundo é constituído pelos catadores, já que podem ficar expostos a potenciais tipos de contaminação presentes nos resíduos. Como consequência de um acidente com material perfurocortante contaminado existe a possibilidade de contaminação por agentes causadores de doenças e patógenos transmitidos pelo sangue ${ }^{6}$.

Neste contexto, faz-se necessário o desenvolvimento educacional, administrativo e até de recursos legislativos para o gerenciamento dos RSS produzidos em ambiente domiciliar. É evidente a necessidade de um plano de ação na prevenção de acidentes e na preservação ambiental ${ }^{7}$.

O objetivo desta pesquisa foi caracterizar e quantificar os resíduos perfurocortantes gerados por diabéticos insulinodependentes que frequentam 15 unidades de saúde da família do município de Umuarama, visando obter informações a respeito do manejo desse material em domicílio, assim como o grau de conhecimento dos geradores sobre a destinação ambientalmente adequada.

\section{Metodologia}

Pesquisa de abordagem quantitativa realizada com diabéticos insulinodependentes que frequentavam 15 unidades de saúde do município de Umuarama (PR). Optou-se pelas unidades que possuem o Programa de Saúde da Família (PSF), já que este assegura a prestação e assistência integral à população na própria instituição de saúde, através de consultas médicas, exames, retirada de medicamentos e atendimento domiciliar. Assim, as unidades selecionadas para esta pesquisa correspondem à integralidade das Unidades Básica de Saúde da Família (UBSF) do município sede, ou seja, 15 UBSF.

A população foi obtida por meio do levantamento nos registros do PSF de cada unidade, com auxílio dos agentes comunitários de saúde. Como critério de inclusão, optou-se pelos usuários que participavam do programa e recebiam assistência domiciliar pelos agentes, totalizando 175 indivíduos.

A pesquisa buscou identificar como esses portadores de diabetes mellitus realizavam o manejo dos resíduos perfurocortantes utilizados em sua terapêutica domiciliar e o grau de informação que estes possuíam a respeito do gerenciamento. Para a coleta de dados foi utilizado um questionário, composto por 21 questões contendo a identificação dos diabéticos (idade, sexo, grau de escolaridade); quantificação e caracterização dos resíduos (número e tipo de seringas, agulhas e lancetas utilizadas por dia); manejo (acondicionamento, descarte); e outros aspectos que envolvam conhecimentos e informações sobre os RSS. Os dados foram coletados no período de 2013 a 2014, em duas etapas: reuniões de Hiperdia, realizada nos postos de saúde, e visita domiciliar.

A primeira etapa, realizada em um período de 6 meses, consistiu-se em visitas a reuniões de Hiperdia - onde são efetuados exames e entrega de medicamentos para diabéticos e hipertensos - 
variando data e local em cada unidade de saúde. Com o auxílio da equipe de enfermagem foram identificados os usuários de insulina presentes no local. Posteriormente, foi realizado um levantamento dos diabéticos que não compareceram à reunião e foram feitas visitas domiciliares com a presença dos agentes de saúde. Houve uma redução de 26 indivíduos da população (que não compareceram à reunião e não estavam em domicílio), previamente selecionada para o estudo. Assim, a amostra foi composta por $149(85,14 \%$ dos pacientes com DM) usuários de insulina.

Utilizou-se o teste Qui-quadrado para verificar a associação entre as variáveis: "conhecimento e descarte", "descarte e acondicionamento" e "descarte e informação", por meio do teste de independência do Qui-quadrado em tabelas de contingência. O nível de significância adotado foi de $1 \%(\mathrm{p}<0,01)$.

\section{Resultados}

Conforme a Tabela 1, dos 149 diabéticos que participaram da pesquisa, $65,77 \%$ pertenciam ao sexo feminino; $61,07 \%$ eram idosos com mais de 61 anos e 50,34\% não haviam concluído o ensino fundamental. Pacientes com diabetes tipo 2 prevaleceram com $88,59 \%$ dos casos. Já em relação ao tempo de diagnóstico, a maioria dos indivíduos $(82,55 \%)$ declarou ter identificado a doença há mais de cinco anos. O principal local de aquisição do material perfurocortante foi a Central Farmacêutica (CF) do município referida por $88,59 \%$ dos entrevistados.

A maioria dos usuários afirmou utilizar como ferramenta para a aplicação de insulina a seringa acoplada com agulha, no entanto, notou-se que $11,49 \%$ fazem uso de canetas injetáveis reutilizáveis, descartando as agulhas separadamente.

Notou-se ainda (Tabela 2) que a maioria dos entrevistados utiliza uma agulha ao dia. A reutilização das agulhas é frequente entre os pacientes, independentemente dos dispositivos de injeção (seringa ou caneta), sendo uma única agulha utilizada mais de 2 vezes.

Quanto ao monitoramento glicêmico, $85,23 \%$ dos entrevistados o realizam em casa, e alguns destes reutilizam as lancetas antes de descartá-las. Ao avaliar a quantidade mensal de lancetas utilizadas verificou-se o seguinte resultado: $53,69 \%$ dos depoentes utilizam mais de $10 ; 20,13 \%$ usam de 1 a 4 e $10,74 \%$ usam de 5 a 10 unidades.

É importante evidenciar que dos 149 entrevistados apenas $11,41 \%$ alegaram não fazer o
Tabela 1. Distribuição dos diabéticos insulinodependentes, de acordo com o gênero, faixa etária, grau de escolaridade, tempo de diagnóstico e tempo da doença, local de aquisição do material. Município de Umuarama-PR, 2013-2014.

\begin{tabular}{|c|c|c|}
\hline Variável & n. & $\%$ \\
\hline \multicolumn{3}{|l|}{ Gênero } \\
\hline Feminino & 98 & 65,77 \\
\hline Masculino & 51 & 34,23 \\
\hline \multicolumn{3}{|l|}{ Idade } \\
\hline Até 10 anos & 5 & 3,36 \\
\hline 11 a 20 anos & 4 & 2,68 \\
\hline 21 a 30 anos & 2 & 1,34 \\
\hline 31 a 40 anos & 10 & 6,71 \\
\hline 41 a 50 anos & 9 & 6,04 \\
\hline 51 a 60 anos & 28 & 18,79 \\
\hline Acima de 61 anos & 91 & 61,07 \\
\hline \multicolumn{3}{|l|}{ Grau de escolaridade } \\
\hline Analfabeto & 35 & 23,49 \\
\hline Ensino fundamental incompleto & 75 & 50,34 \\
\hline Ensino fundamental completo & 9 & 6,04 \\
\hline Ensino médio incompleto & 6 & 4,03 \\
\hline Ensino médio completo & 21 & 14,09 \\
\hline Ensino superior & 3 & 2,01 \\
\hline \multicolumn{3}{|l|}{ Tipo de diabetes } \\
\hline Tipo 1 & 17 & 11,41 \\
\hline Tipo 2 & 132 & 88,59 \\
\hline \multicolumn{3}{|l|}{ Tempo de diagnóstico da doença } \\
\hline A 1 ano & 3 & 2,01 \\
\hline 2 a 5 anos & 23 & 15,44 \\
\hline Mais de 5 anos & 123 & 82,55 \\
\hline \multicolumn{3}{|l|}{ Local de aquisição do material } \\
\hline Farmácia & 17 & 11,41 \\
\hline Central Farmacêutica & 132 & 88,59 \\
\hline
\end{tabular}

* n. de cada variável: 149 .

teste glicêmico em residência. Muitos indivíduos disseram não estar recebendo as fitas reagentes distribuídas de forma gratuita, admitindo não ter condições financeiras para comprá-las. Fato que interfere diretamente na quantidade de resíduos gerados neste período.

Ao serem interrogados sobre o acondicionamento dos resíduos (Tabela 3), 46,98\% dos indivíduos afirmaram acondicionar seu material dentro de algum recipiente, como: garrafa PET e de água, frasco de maionese e de remédio, caixa de papelão e de isopor, e o descarpack. A utilização de caixas próprias para o descarte do material perfurocortante (descarpack) foi referida por apenas dois dos entrevistados, que dizem comprá -las. Em contrapartida, um número considerável 
Tabela 2. Distribuição da quantidade de seringas acopladas com agulhas e quantidade de reutilização destas na aplicação de insulina. Município de Umuarama - PR, 2013-2014.

\begin{tabular}{lrr}
\hline \multicolumn{1}{c}{ Variável } & n. & \multicolumn{1}{c}{$\%$} \\
\hline *Quantidade de seringas acopladas & & \\
com agulhas utilizadas por dia & & \\
Uma ao dia & 117 & 88,64 \\
2 a 3 ao dia & 8 & 6,06 \\
1 a cada 2 dias & 3 & 2,27 \\
1 a cada 3 dias & 3 & 2,27 \\
Frequência não definida & 1 & 0,76 \\
* Reutilização das seringas acopladas & & \\
Não reutiliza & 44 & 33,33 \\
2 a 3 vezes & 56 & 42,42 \\
Mais de 3 vezes & 31 & 23,49 \\
Frequência não definida & 1 & 0,76 \\
** Quantidade diária de agulhas & & \\
utilizadas nas canetas & & \\
Uma & 11 & 64,71 \\
2 a 3 & 5 & 29,41 \\
Mais de 3 & 1 & 5,88 \\
** Reutilização das agulhas utilizadas & & \\
nas canetas & & \\
1 vez & 1 & 5,88 \\
2 a 3 vezes & 3 & 17,65 \\
Mais de 3 vezes & 7 & 41,18 \\
Não respondeu & 6 & 35,29 \\
\hline n. de cada variável: 132; ${ }^{* * ~ n . ~ d e ~ c a d a ~ v a r i a ́ v e l: ~}$ & 17. & \\
& &
\end{tabular}

Tabela 3. Acondicionamento e descarte dos resíduos perfurocortantes gerados pela aplicação de insulina e monitoramento da glicemia capilar. Município de Umuarama - PR, 2013-2014.

\begin{tabular}{lrr}
\hline \multicolumn{1}{c}{ Variável } & n. & \multicolumn{1}{c}{$\%$} \\
\hline Acondicionamento & & \\
Joga sem nenhuma proteção & 30 & 20,13 \\
Envolve em papel & 14 & 9,40 \\
Coloca dentro de algum recipiente & 70 & 46,98 \\
Sacos plásticos & 31 & 20,80 \\
Outros & 4 & 2,69 \\
Descarte & & \\
Descarta no meio ambiente & 2 & 1,34 \\
Descarta no lixo comum & 79 & 53,02 \\
Descarta no lixo reciclável & 6 & 4,03 \\
Descarta no posto de saúde & 34 & 22,82 \\
Descarta na Central F./farmácia & 6 & 4,03 \\
Descarta em fossa asséptica & 5 & 3,35 \\
Não realiza o descarte (casa) & 9 & 6,04 \\
Enterra & 1 & 0,67 \\
Outros & 7 & 4,70 \\
\hline * de cada variável: 149. & &
\end{tabular}

de pessoas afirmou não acondicionar seus resíduos em recipientes específicos e, consequentemente, descartá-los sem nenhuma proteção.

A maioria dos depoentes afirmou descartar seus resíduos perfurocortantes de forma incorreta (Tabela 3), sendo que 53,02\% relatou como destino o lixo comum.

No tocante as orientações recebidas referentes ao descarte, $44,97 \%$ afirmaram tê-las adquiridas por meio do posto de saúde, médico, enfermeiro, agente comunitário, trabalho e TV. Por outro lado, 55,03\% dos indivíduos disseram não ter recebido qualquer informação a respeito do assunto e $88,59 \%$ declararam não conhecer um programa de coleta de RSS.

Embora muitos diabéticos atestaram não ter recebido orientações relacionadas ao descarte dos resíduos, verificou-se que $60,80 \%$ possuem ciência dos riscos inerentes a práticas incorretas de disposição final, como pode-se observar nos seguintes comentários: "Contamina o lixo"; "Pode machucar alguém"; "As pessoas podem se furar, contaminação"; "O lixeiro pode se furar"; "Machucar as pessoas"; "Infecção de quem meche com o lixo"; "Deve ser muito ruim, faz parte do lixo do hospital"; "Perigoso, poluente, danos na natureza"; "Afeta o ambiente"; "O lixeiro pode se cortar, drogados roubar a seringa"; "O reciclável vai separar e destinar ao lixo hospitalar"; "Machucar, furar igual vidro quebrado"; "Transmite doença, não pode se descartado no lixo comum”.

Finalmente, ao serem interrogados sobre o conhecimento da norma RDC no 306/04, que versa sobre o manejo dos RSS no Brasil, apenas 6,04\% dos diabéticos afirmaram já conhecê-la, obtendo as informações por meio da televisão; posto de saúde e médico.

Para melhor compreensão da relação entre as principais variáveis investigadas, foram realizadas correlações entre o modo de descarte correto/incorreto e as seguintes categorias: $1^{\mathrm{a}}$ "Acondicionamento correto/incorreto"; 2a "Recebeu informação referente ao descarte correto/não recebeu informação" e $3^{\text {a } ~ " p o s s u i ~ c o n h e c i m e n t o ~}$ dos riscos inerentes ao descarte incorreto/não possui conhecimentos".

As respostas permitiram concluir que maioria das pessoas que descarta seu material de maneira incorreta também o acondiciona de forma inadequada. Analisando estes dados estatisticamente, houve diferença significativa $\left(\mathrm{p}<2,78 \times 10^{-5}\right)$ entre a forma de acondicionamento e o descarte ao nível de significância de $1 \%$.

$\mathrm{Na}$ segunda categoria, informação, verificouse que a maioria das pessoas que relatou não ter 
recebido informação descarta seu material de forma incorreta. Assim, os dados revelam estatisticamente que o descarte correto/incorreto parece depender da presença/ausência de informação ao nível de significância de $1 \%,\left(\mathrm{p}<2,52 \times 10^{-5}\right)$.

$\mathrm{Na}$ terceira categoria, conhecimento, observou-se que a maioria dos indivíduos que atestou não possuir conhecimento a respeito do descarte dos resíduos perfurocortantes afirmou descartar seu material de forma incorreta. As análises estatísticas também demonstraram que as categorias não são uniformes, ou seja, há diferença estatisticamente significante entre a variável "descarte correto/incorreto dos resíduos" e o conhecimento a respeito desta prática, ao nível de significância de $1 \%\left(\mathrm{p}<5,76 \times 10^{-5}\right)$.

\section{Discussão}

A maioria dos entrevistados do presente estudo era idosos e possuía o diabetes tipo 2 (DM2), que acomete, principalmente, em adultos com mais de 40 anos $^{8}$. Estima-se que haverá um acréscimo de $50 \%$ do número de casos mundiais até o ano de $2025^{\circ}$, crescimento que pode estar relacionado ao envelhecimento da população e à obesidade ${ }^{10}$. Ademais, constatou-se a prevalência do gênero feminino dentre os entrevistados, o que pode estar relacionado ao fenômeno de feminização, ou seja, à maior expectativa de vida entre as mulheres em relação aos homens ${ }^{11} \mathrm{e}$ à procura das mulheres aos serviços de saúde, favorecendo o diagnóstico mais precoce.

Houve também um baixo nível de escolaridade entre os participantes. Esse cenário foi semelhante aos encontros de outros autores que investigavam pacientes com DM2 no Brasil ${ }^{12-14}$. É importante ressaltar que a baixa escolaridade é uma das características apresentada pela população atendida pelo Sistema Único de Saúde (SUS). Desse modo, torna-se necessária a adoção de estratégias educacionais que possibilite a esses indivíduos o acesso à informação, relacionada ao cuidado com a saúde e ao descarte dos resíduos gerados.

Entre os resíduos gerados em domicílio pelos pacientes com DM, estavam seringas, agulhas e lancetas. Na literatura também se pôde observar a frequente utilização e descarte desses materiais pelos diabéticos. Em Nova Deli, na Índia, um estudo realizado no ano de 2011 com 303 indivíduos divulgou o descarte semanal de perfurocortantes, onde $71 \%$ dos participantes desprezavam pelo menos 7 agulhas e $89 \%$ ao menos 7 lancetas/ semana ${ }^{15}$. Na Inglaterra, uma pesquisa no distrito de South Staffordshire, estimou que aproximadamente 2,5 milhões de resíduos foram gerados anualmente por pacientes diabéticos e mais da metade desses foram descartados junto ao lixo doméstico ${ }^{16}$.

Diante do número expressivo de geração e descarte desses resíduos, os usuários de insulina têm adotado como prática a reutilização do material antes de descartá-lo, o que é comumente encontrado na literatura ${ }^{3,17-19}$. É importante ressaltar que o Ministério da Saúde admite o reuso das seringas com agulhas acopladas por até oito aplicações ${ }^{20}$. Logo, o processo de reutilização de seringas está diretamente associado à quantidade de resíduos gerados em domicílio ${ }^{21}$.

A partir das respostas obtidas com o número de aplicações e de reuso dos materiais, realizou-se uma estimativa da quantidade anual de resíduos descartados pelos participantes deste estudo, quais sejam: 33.324 seringas (com agulha), 2.772 unidades agulhas (utilizadas nas canetas) e 39.558 lancetas. Se não bastasse esse grande número, convém registrar também que mais da metade dos investigados relataram descartar seus resíduos em locais impróprios, sendo o lixo domiciliar o mais comum. Esse modo também é reconhecido por outras pesquisas realizadas não só no Brasil, mas em outros países, como Índia, Inglaterra, França, Luxemburgo, Bélgica e Suíç̧⿻ 3,15-19,22.

Em decorrência do descarte incorreto, há muitos registros na literatura envolvendo acidentes com resíduos cortantes (com sangue e outros fluidos orgânicos) e possível presença de agente infectante. Os profissionais envolvidos são tanto o pessoal da atenção à saúde como o da limpeza e coleta dos resíduos. No Rio de Janeiro, pesquisa revelou que os resíduos perfurocortantes estão entre as causas mais importantes de acidentes com coletores de lixo domiciliar ${ }^{23}$.

Diante dos riscos inerentes a esses resíduos é fundamental que eles sejam armazenados em recipientes rígidos e resistentes à punctura e, logo após o seu preenchimento, sejam encaminhados à UBS para que a mesma faça o descarte adequado ${ }^{20}$.

Para isso, é necessário que sejam apresentadas à população diferentes formas de acondicionamento, considerando que existem alternativas seguras $^{21}$. No estudo foram encontradas algumas soluções improvisadas, como garrafa PET, frasco de amaciante e de maionese. Contudo, notou-se que muitos indivíduos admitiram utilizar sacolas plásticas, as quais apresentam características totalmente desfavoráveis para acondicionar resíduos cortantes. 
Finalmente, os achados desse estudo mostram que a forma de acondicionamento e descarte dos resíduos está intrinsecamente relacionada às orientações recebidas. A prova disso é o resultado obtido na correlação entre as "orientações" $\mathrm{e}$ "descarte" a qual revelou que a maioria daqueles que não recebeu informação sobre o manejo dos resíduos os desprezavam de maneira inadequada. É oportuno informar que a falta de informação também foi encontrado em outros estudos ${ }^{17,18,24}$, como o de um distrito da Inglaterra que concluiu que os diabéticos que não lembraram de receber conselhos foram mais propensos a descartar seus materiais cortantes de maneira insegura ${ }^{16}$. No Ceará os resultados de uma pesquisa revelaram que os pacientes que foram instruídos sobre o manejo adequado dos RSS são 21 vezes mais propensos a descartá-los corretamente ${ }^{13}$.

Portanto, faz-se primordial o estabelecimento de estratégias de educação em saúde no tratamento de pessoas com DM, de modo que elas integrem o aconselhamento sobre a doença, cabendo à equipe multidisciplinar a responsabilidade de educar e reforçar a informação sobre os métodos corretos de eliminação de resíduos ${ }^{13}$.

\section{Conclusão}

Os resultados obtidos nesse estudo permitem concluir que a ausência de orientações sobre o descarte correto de perfurocortantes aos pacientes insulinodependentes faz com que esses resíduos acabem sendo desprezados em meio aos resíduos comuns, proporcionando potenciais riscos à saúde pública e ao meio ambiente. Com base no diagnóstico realizado, é possível traçar uma estratégia para gestão desses materiais, abrangendo o armazenamento em domicílio, até o descarte na unidade de saúde. Neste contexto, cabe às autoridades municipais desenvolver um programa de gestão ambiental que abranja ações a curto, médio e longo prazo. Vale ressaltar, que a educação ambiental é o principal instrumento para a conscientização e mudança de hábitos da população, devendo esta ser realizada de forma contínua. Outro aspecto importante a se considerar, é a capacitação profissional, pois os profissionais das unidades de saúde precisam estar preparados para orientar os pacientes e a comunidade em seu entorno sobre as formas de manejo dos RSS.

\section{Colaboradores}

LN Santos contribuiu com a coleta e análise dos dados e redação do manuscrito. JB Ruiz é orientadora do trabalho e foi responsável pelo delineamento da pesquisa, escolha da metodologia e revisão final da redação. 


\section{Referências}

1. Brasil. Agência Nacional de Vigilância Sanitária (ANVISA). Resolução RDC n 306, de 7 de dezembro de 2004. Dispõe regulamento técnico para o gerenciamento de resíduos de serviços de saúde. Diário Oficial da União; 2004.

2. Brasil. Ministério da Saúde (MS). Caderno de Atenção Básica. Diabetes mellitus. Brasília: MS; 2006.

3. Adel RVC, Grossi SAA. Reutilização de seringas descartáveis no domicílio de crianças e adolescentes com diabetes mellitus. Rev Esc Enferm USP 2007; 41(2):187-195.

4. Vaz KV, Freitas MM, Cirqueira JZ. Investigação sobre a forma de descarte de medicamentos vencidos. Cenarium Pharmacêutico 2011; 4(4):3-27.

5. Ferreira JÁ, Anjos LA. Aspectos de saúde coletiva e ocupacional associados à gestão dos resíduos sólidos municipais. Cad Saúde Pública 2001; 17(3):689-696.

6. Marziale MHP, Nishimura KYN, Ferreira, MM. Riscos de contaminação ocasionados por acidentes de trabalho com material pérfuro-cortante entre trabalhadores de enfermagem. Rev Latino-Am Enfermagem 2004; 12(1):36-42.

7. Vieira M, Padilha MICS. O HIV e o trabalhador de enfermagem frente ao acidente com material perfurocortante. Rev Esc Enferm USP 2008; 42(4):804-810.

8. Silva ARV, Zanetti ML, Forti AC, Freitas WJF, Hissa MN, Damasceno MMC. Avaliação de duas intervenções educativas para a prevenção do diabetes mellitus tipo 2 em adolescentes. Texto Contexto Enferm 2011; 20(4):782-787.

9. Boyle JP, Honeycutt AA, Narayan KMV, Hoerger TJ, Gleiss LS, Chen H, Thompson TJ. Projection of Diabetes Burden Through 2050. Diabetes Care 2001; 24(11):1936-1940.

10. Viegas K. Prevalência de diabetes mellitus na população de idosos de Porto Alegre e suas características sociodemográficas e de saúde [tese]. Porto Alegre: Pontifícia Universidade Católica do Rio Grande do Sul; 2009.

11. Organização Mundial da Saúde (OMS). Envelhecimento Ativo: Uma Política de Saúde. Brasília: OPAS; 2005.

12. Goldenberg P, Schenkman S, Franco LJ. Prevalência de diabetes mellitus: diferenças de gênero e igualdade entre os sexos. Rev Bras Epidemiol 2003; 6(1):18-28.

13. Cunha GHD, Barbosa RVA, Fontenele MSM, Lima MAC, Franco KB, Fechine FV. Insulin therapy waste produced in the households of people with diabetes monitored in Primary Care. Rev Bras Enferm 2017; 70(3):618-625.

14. Otero LM, Zanetti ML, Ogrizio MD. El conocimiento del paciente diabético sobre su enfermedad, antes y después de la inplementación de um programa de educación em diabetes. Rev Latino-Am Enfermagem 2008; 16(2):221-237.
15. Singh AP, Chapman R.S. Knowledge, Attitude and Practices (KAP) on Disposal of Sharp Waste, Used for Home Management of Type-2 Diabetes Mellitus, in New Delhi, India. J Health Res 2011; 25(3):135-140.

16. Olowokure B, Duggal H, Armitage L. The disposal of used sharps by diabetic patients living at home. Int $J$ Environ Health Res 2003; 13(2):117-123.

17. Stacciarini TSG, Pace AE, Iwamoto HH. Distribuição e utilização de seringas para aplicação de insulina na Estratégia Saúde da Família. Rev Eletr Enf 2010; 12(1):47-55.

18. Silva ÉNSF, Santana OS, Palmeira CS. Descarte de Seringas e agulhas por pacientes com diabetes mellitus. Rev Enferm Contemporânea 2013; 2(1):82-102.

19. Teixeira CRS, Zanetti ML, Ribeiro KP. Reutilização de seringas descartáveis: frequência e custos para administração de insulina no domicílio. Rev Latino-Am Enferm 2001; 9(5):47-54.

20. Brasil. Ministério da Saúde (MS). Cadernos de Atenção Básica. Estratégias para o cuidado da pessoa com doença crônica. Brasília: MS; 2013.

21. Pinto ARC, Fonseca SX, Santana TG, Moraes TA, Souza UF. O manejo e descarte do lixo biológico no ambiente intradomiciliar: uma prática diária vivenciada pelo diabético insulinodependente. Rev Rede Cuid Saúde 2011; 5(1):1-23.

22. Bouhanick B, Hadjadj S, Weekers L. What to the needls, syringes, lancets and reagente strips of diabetic patients become in the absence of a common atitude? About 1070 questionnaires in diabetic clinics. Diabetes Metab 2000; 26(4):288-293.

23. Velloso MP, Santos EM, Anjos LA. Processo de trabalho e acidentes de trabalho em coletores de lixo domiciliar na cidade do Rio de Janeiro, Brasil. Cad Saúde Pública 1997; 13(4):693-700.

24. Tapia CEV. Diabetes Mellitus e o descarte de seringas e agulhas. Rev Gaúcha Enferm 2009; 30 (2):228-34.

Artigo apresentado em 16/04/2018

Aprovado em 11/10/2018

Versão final apresentada em 13/10/2018 
